\title{
YOUNG STARS FAR FROM THE GALACTIC PLANE: RUNAWAYS FROM CLUSTERS
}

\author{
Christine Allen $^{1}$ and T. D. Kinman ${ }^{2}$ \\ RESUMEN
}

En fechas recientes se ha encontrado un número significativo de estrellas $\mathrm{OB}$ lejos del plano galáctico, situadas a distancias entre algunos cientos de pc y varios kpc. La corta vida de estas estrellas plantea problemas para su interpretación en el marco usual de la formación estelar. Se han propuesto varios mecanismos para explicar la existencia de estas estrellas, tanto desde el punto de vista convencional, o bien postulando formación estelar en el halo mismo. Los mecanismos convencionales varían desde argumentar que se trata de estrellas mal clasificadas, y por ende, cercanas y poco masivas, hasta postular potentes mecanismos para su expulsión del plano galáctico. Las explicaciones que postulan formación in situ también tienen variantes. Hemos compilado de la literatura una lista de estrellas jóvenes lejos del plano, para las cuales parece convincente la evidencia de su juventud. Discutimos dos posibles mecanismos de formación para estas estrellas: expulsión del plano como resultado de la evolución dinámica de cúmulos estelares pequeños (Poveda et al. 1967) y formación in situ a través de choques inducidos por ondas espirales de densidad (Martos et al. 1999). Calculamos órbitas galácticas para estas estrellas e identificamos aquellas que pueden explicarse por uno u otro mecanismo. Concluimos que aproximadamente el 90 por ciento de ellas pueden ser explicadas por el mecanismo de expulsión de cúmulos, es decir, pueden identificarse como estrellas desbocadas en el halo galáctico.

\begin{abstract}
Quite recently, a significant number of $O B$ stars far from the galactic plane have been found, situated at $z$ distances ranging from several hundreds of pc to several kpc. The short lifetimes of these stars pose problems for their interpretation in terms of the standard picture of star formation. Different mechanisms have been put forward to explain the existence of these stars, either within the conventional view, or postulating star formation in the galactic halo itself. These mechanisms range from arguing that they are misidentified evolved or abnormal stars, to postulating powerful ejection mechanisms for young disk stars; in situ formation also admits several variants. We have collected from the literature a list of young stars far from the plane, for which the evidence of youth seems convincing. We discuss two possible formation mechanisms for these stars: ejection from the plane as the result of dynamical evolution of small clusters (Poveda et al. 1967) and in situ formation, via induced shocks created by spiral density waves (Martos et al. 1999). We compute galactic orbits for these stars, and identify the stars that could be explained by one or the other mechanism. We find that about 90 percent of the stars can be accounted for by the cluster ejection mechanism, that is, they can be regarded as runaway stars in the galactic halo.
\end{abstract}

Key Words: STARS: EARLY-TYPE - STARS: KINEMATICS

\section{INTRODUCTION}

There exists an anomalous group of early-type - hence mostly young - stars located far from the galactic plane, at $z$-distances ranging from one to more than $10 \mathrm{kpc}$. First studied were the A-type stars (Rodgers et al. 1981; Lance 1988). Such intermediate-age stars have most recently been studjed by Preston and Sneden (2000) and are likely to be blue stragglers. More recently, a number of $\mathrm{OB}$

\footnotetext{
${ }^{1}$ Instituto de Astronomia, Universidad Nacional Autonoma de Mexico.

${ }^{2}$ Kitt Peak National Observatory, National Optical Astronomy Observatory.
}

stars have been found, also situated at vertical distances of up to several kpc from the plane (Conlon et al. 1989; Conlon et al. 1990; Conlon 1992; Schmidt et al. 1997; Ringwald et al. 1998). The shorter lifetimes of these stars aggravate the problems of their interpretation within the standard picture of star formation and galactic evolution. The most extreme examples, if they originated in the plane, must have been ejected with velocities surpassing $1000 \mathrm{~km} \mathrm{~s}^{-1}$, which clearly are unrealistically high values.

Different mechanisms have been put forward to explain the existence of these stars, either within the conventional view, or postulating star formation in 
the galactic halo itself (see for instance Lance 1988; or Tobin 1991, for extensive reviews). These mechanisms range from arguing that they are misidentified evolved or pathological stars, to postulating powerful ejection mechanisms for young, thin disk stars, or to proposing that they were formed in situ, in the galactic halo, from a mixture of gas acquired while the Galaxy captured a small satellite galaxy, or from collisions between cloudlets, or other possibilities.

It is now clear that the anomalous stars are quite a mixed bag themselves, and that, as a group, they undoubtedly contain some misidentified evolved stars of Population I, or some Population II stars posing as young B stars. But the youth of quite a number of them seems well established, as shown by accurate determinations of surface gravities and colors, high resolution spectral studies, detailed abundance determinations, rotational velocities, etc. Among the genuinely young OB stars far from the plane, it is also clear that no single mechanism is capable of explaining all cases. Extreme examples, like PG 1002+506, a Be star with $z>10 \mathrm{kpc}$ (Ringwald et al. 1998), PG 0009+036, a rapidly rotating normal B star at $z>5 \mathrm{kpc}$ (Schmidt et al. 1996), and others, are likely to remain puzzling for the foreseeable future. Nonetheless, Tobin (1991) concludes that dynamical ejection from small clusters in the plane, as proposed for ordinary runaway stars by Poveda et al. (1967) and further studied by Gies end Bolton (1986), Leonard and Duncan (1988, 1990), and others, remains the most likely explanation for many of the young stars at large distances from the plane. Clearly, it is then of importance to determine for which stars this mechanism is the likely explanation.

\section{A LIST OF YOUNG STARS FAR FROM THE GALACTIC PLANE}

Although there are many more examples of presumably young stars far from the galactic plane scattered in the literature, for the purposes of this work we will focus our attention on two groups of stars. One is the relatively homogeneous group of 32 stars studied by Conlon et al. (1990). The evidence for the youth of these stars comes from detailed, high resolution abundance studies not only of elements of the CNO group, but also of heavier elements, notably $\mathrm{Al}$ and $\mathrm{Fe}$. These determinations allow the authors to conclude that their stars are bona fide, normal young B stars, and not evolved, intermediate composition stars, or Population II stars mimicking the spectroscopic characteristics of early type stars. The second group is composed of ten stars, with data by

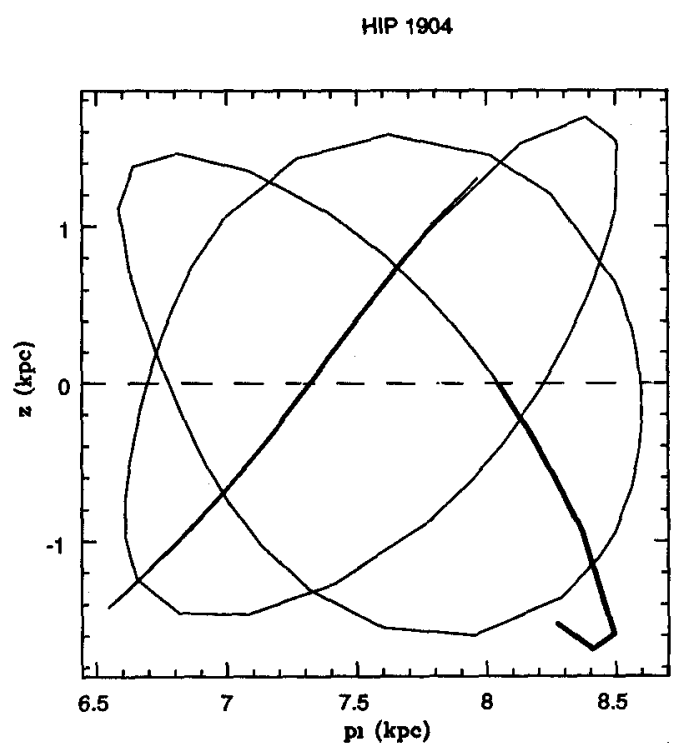

Fig. 1. The galactic orbit of HIP 1904. The figure shows the meridional orbit, that is, the instantaneous projection of the three-dimensional trajectory on the plane that contains the star and the $z$ axis. Only the part of the orbit marked with a thick line, starting at the present position of the star, and ending on the galactic plane, is used to calculate the flight time. A significant error would be introduced if the flight time were calculated assuming a straight-line, vertical trajectory from the galactic plane.

HIP 13800

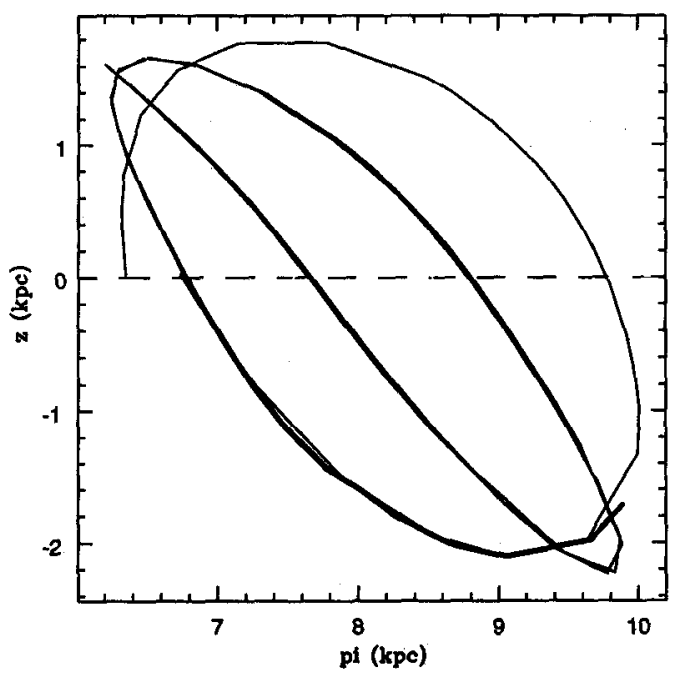

Fig. 2. The meridional galactic orbit of HIP 13800. For details, see caption, Fig. 1. Here again, a time of flight calculated using a straight vertical trajectory would be erroneous 
HIP 112790

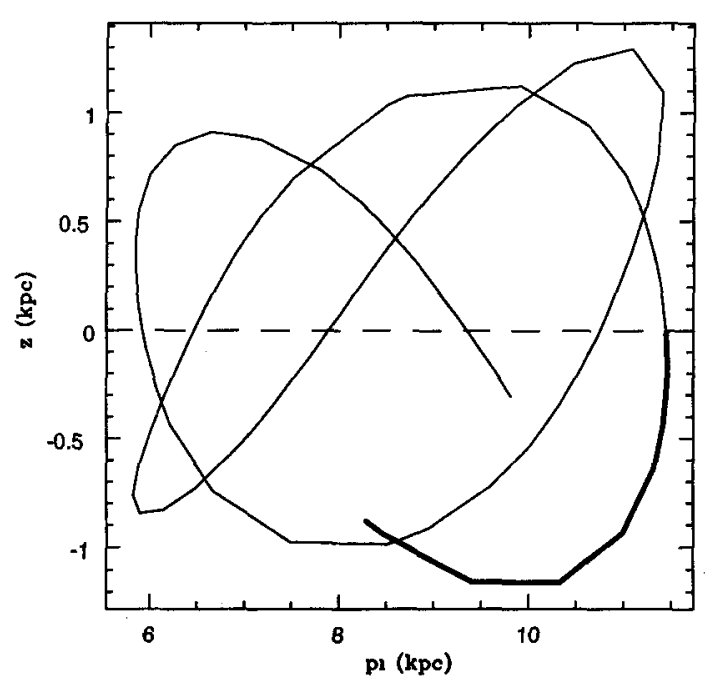

Fig. 3. The galactic orbit of HIP 112790. For details, see caption, Fig. 1. In this case, too, the calculation of the correct time of flight requires knowledge of the actual trajectory of the star.

HIP 107207

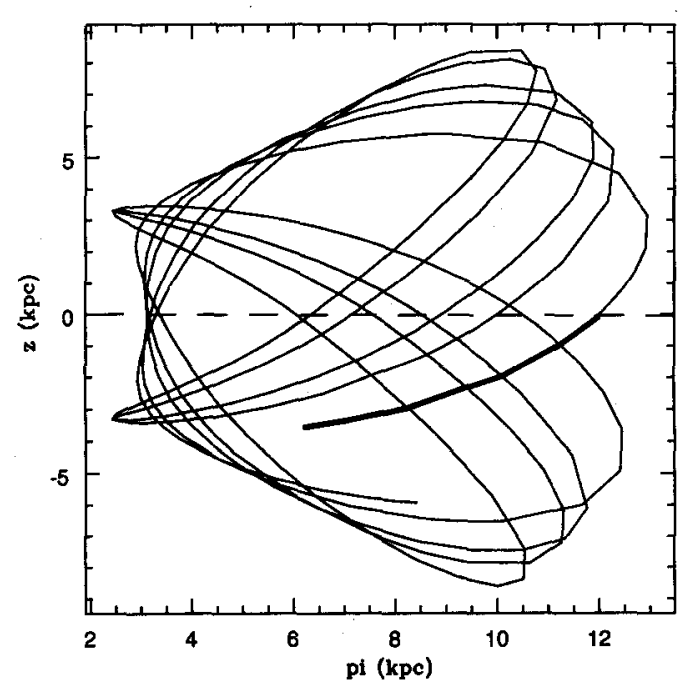

Fig. 4. The galactic orbit of HIP 107207 For details, see caption, Fig. 1, 2 and 3

several observers. Though not as homogeneous as the Conlon et al. list, these stars are also most probably bona fide young stars. They will, however, be treated as a separate group.

\section{ORBITAL ANALYSIS}

\subsection{The Conlon stars}

By means of a rudimentary estimation of the times of flight of these stars, assuming they were dynamically ejected from the plane, Conlon et al. concluded that ejection was indeed the most likely mechanism to explain the majority of them.

We have obtained improved estimates of the times of flight by numerically integrating the galactic orbits of these stars. We have updated the proper motions of the Conlon et al. stars using Hipparcos data. Radial velocities were taken, when available, from the Hipparcos Input Catalogue; otherwise, they were calculated from the data given by Conlon et al. We also adopted their values for the distances. We then proceeded to numerically integrate the galactic orbit of each star. The galactic potential model of Allen and Santillán (1993) was used for the integration of the orbits.

Figures 1 to 4 show examples of the meridional galactic orbits of four stars. All previous estimates of the times of flight for these stars have assumed that the stars' trajectories are perpendicular to the galactic plane, and most authors neglect the gravitational deceleration. Figures 1 to 4 show that the actual trajectories differ considerably from straight vertical lines, and that therefore, the times of flight based on such an approximation are generally longer. For each of the stars, then, actual times of flight since they left the galactic plane were obtained from the orbit computations. These times of flight were compared with the nuclear lifetimes of disk-composition stars using the models of Schaller et al. (1992). The stellar masses determined by Conlon et al. were used. The results of this analysis are summarized in Table 1. Successive columns contain the Hipparcos number of the star, the time elapsed since it left the plane, the mass, the main sequence lifetime, the velocity of ejection from the plane, the estimated error in the computed times of flight, the times of flight if the stars formed at $z=700 \mathrm{pc}$, and finally, a code tagging the stars that can be explained by the ejection mechanism, or some variants thereof.

Table 1 shows that for 24 out of the 32 stars the times of flight are smaller than the nuclear lifetimes. It is clear, then, that these stars could indeed have been ejected from the plane. They are marked by a ' $y$ ' (for yes) in the last column of Table 1. The ejection velocities, also obtained from the orbital analysis, are shown in Column 5. Their values are quite reasonable, and compatible with the ejection model. The errors in the times of flight, shown in Column 6 , were estimated by computing two additional orbits 
TABLE 1

RESULTS: THE CONLON ET AL. STARS

\begin{tabular}{|c|c|c|c|c|c|c|c|}
\hline Star & $\begin{array}{l}t \text { (flight) } \\
10^{6} \mathrm{y}\end{array}$ & $\begin{array}{c}\text { mass } \\
M_{\odot}\end{array}$ & $\begin{array}{l}m \text {-s life } \\
10^{6} \mathrm{y}\end{array}$ & $\begin{array}{l}v-e j \\
\mathrm{~km} \mathrm{~s}^{-1}\end{array}$ & $\begin{array}{c}\operatorname{sigma}(t) \\
10^{6} \mathrm{y}\end{array}$ & $\begin{array}{c}t(700 \mathrm{pc}) \\
10^{6} \mathrm{y}\end{array}$ & ejection \\
\hline HIO01904 & 51.090 & 6 & 63.1 & 93.3 & 5 & & $\mathrm{y}$ \\
\hline HI002702 & 55.530 & 5 & 94.5 & 73.7 & 7 & & $\mathrm{y}$ \\
\hline HI003812 & 49.464 & 9 & 26.4 & 133.8 & 5 & 43 & no \\
\hline HI006419 & 26.584 & 4 & 164.7 & 64.8 & 5 & & $\mathrm{y}$ \\
\hline HI011809 & 12.160 & 3 & 352.5 & 134.4 & 3 & & $\mathrm{y}$ \\
\hline HI012320 & 37.413 & 3 & 352.5 & 68.8 & 14 & & $\mathrm{y}$ \\
\hline HI013800 & 61.676 & 6 & 63.1 & 117.1 & 8 & & $\mathrm{y}$ \\
\hline HI016130 & 40.480 & 4 & 164.7 & 50.7 & 5 & & $\mathrm{y}$ \\
\hline HI016466 & 20.418 & 5 & 94.5 & 40.8 & 4 & & $\mathrm{y}$ \\
\hline HI016758 & 21.069 & 15 & 11.6 & 147.0 & 2 & 12 & $\mathrm{f}$ \\
\hline HI51624AB & 12.139 & 21 & 8.0 & 51.1 & 2 & 0 & $\mathrm{r}, \mathrm{f}$ \\
\hline HI052906 & 54.068 & 8 & 31.6 & 171.0 & 7 & 48 & no \\
\hline HI055051 & 5.612 & 11 & 17.6 & 159.0 & 1 & & $\mathrm{y}$ \\
\hline HI055461 & 39.448 & 5 & 94.5 & 123.2 & 3 & & $\mathrm{y}$ \\
\hline HI058046 & 13.266 & 3 & 352.5 & 88.1 & 5 & & $y$ \\
\hline HI059067 & 48.547 & 5 & 94.5 & 288.8 & 2 & & $y$ \\
\hline HI059160 & 14.710 & 5 & 94.5 & 227.0 & 1 & & $y$ \\
\hline HI059955 & 21.482 & 4 & 164.7 & 61.3 & 3 & & $\mathrm{y}$ \\
\hline HI060615 & 37.639 & 8 & 31.6 & 119.5 & 4 & 30 & $\mathrm{r}, \mathrm{f}$ \\
\hline HI070275 & 13.820 & 10 & 22.4 & 281.0 & 1 & & $y$ \\
\hline HI079649 & 24.550 & 9 & 26.4 & 84.4 & 3 & & $y$ \\
\hline HI105912 & 16.585 & 10 & 22.4 & 140.0 & 2 & & $\mathrm{y}$ \\
\hline HI107027 & 39.930 & 14 & 12.6 & 212.9 & 7 & 33 & no \\
\hline HI108215 & 36.132 & 5 & 94.5 & 101.0 & 3 & & $y$ \\
\hline HI111396 & 40.060 & 4 & 164.7 & 64.1 & 5 & & $\mathrm{y}$ \\
\hline HI111563 & 19.605 & 15 & 11.6 & 228.0 & 2 & 14 & $\mathrm{f}$ \\
\hline HI112790 & 60.498 & 4 & 164.7 & 88.1 & 8 & & $y$ \\
\hline HI1 13735 & 13.903 & 9 & 26.4 & 161.0 & 1 & & $\mathrm{y}$ \\
\hline HI114569 & 8.316 & 6 & 63.1 & 350.7 & 1 & & $y$ \\
\hline HI114690 & 12.015 & 19 & 8.6 & 157.4 & 1 & 8 & $\mathrm{r}, \mathrm{f}$ \\
\hline HI115347 & 21.675 & 9 & 26.4 & 58.1 & 4 & & $\mathrm{y}$ \\
\hline HI115729 & 24.607 & 8 & 31.6 & 76.7 & 3 & & $\mathrm{y}$ \\
\hline
\end{tabular}

for each star, with the initial conditions modified by the observational errors in distances, proper motions and radial velocities. The uncertainties in the times of flight are fairly small, and are largely due to the estimated errors in the distances.

\subsection{Other stars}

A literature search allowed us to obtain enough data for a further group of 10 stars. Six of these stars were taken from a study of ten young massive B stars (Ramspeck et al. 2001), the other four having no information on proper motions. The remaining stars come from different sources (Tenjes et al. 2001, Conlon et al. 1992, Keenan 1986, Keenan 1981).

The orbital analysis was performed in the same way as for the Conlon stars. The results are displayed in Table 2. Again, we see that the ejection mechanism provides a likely explanation for six out of the ten stars. They are marked with a ' $y$ ' in the 
TABLE 2

RESULTS: OTHER STARS

\begin{tabular}{|c|c|c|c|c|c|c|c|}
\hline Star & $\begin{array}{l}t \text { (flight) } \\
10^{6} \mathrm{y}\end{array}$ & $\begin{array}{c}\text { mass } \\
M_{\odot}\end{array}$ & $\begin{array}{l}m \text {-s life } \\
10^{6} \mathrm{y}\end{array}$ & $\begin{array}{l}v-e j \\
\mathrm{~km} \mathrm{~s}^{-1}\end{array}$ & $\begin{array}{c}\operatorname{sigma}(t) \\
10^{6} \mathrm{y}\end{array}$ & $\begin{array}{c}t(700 \mathrm{pc}) \\
10^{6} \mathrm{y}\end{array}$ & ejection \\
\hline PG0122+214 (1) & 51.0 & 7 & 47.3 & 181.5 & 16 & 45 & $\mathrm{y}$ \\
\hline PG1533+467 (1) & 20.2 & 6 & 63.1 & 253.5 & 2 & 15 & $\mathrm{y}$ \\
\hline PHL346 ${ }^{(1)}$ & 27.9 & 10 & 22.4 & 462.4 & 2 & 25 & $r, f$ \\
\hline BD-15 115 & 31.0 & 8 & 31.6 & 277.7 & 1 & 28 & $\mathrm{y}$ \\
\hline PG1610+239 (1) & 219.7 & 6 & 63.1 & 213.8 & 81 & 205 & no \\
\hline PG2219+094 (1) & 43.5 & 9 & 31.6 & 193.6 & 20 & 40 & $\mathrm{y}$ \\
\hline HI060350 & 20.4 & 5 & 94.5 & 418.8 & 2 & 17 & $\mathrm{y}$ \\
\hline $\mathrm{BD}-23766^{(3)}$ & 15.3 & 10 & 22.4 & 423.9 & 2 & 13 & $\mathrm{y}$ \\
\hline HD18100 (4) & 22.3 & 17 & 10.1 & 186.1 & 3 & 15 & $r, f$ \\
\hline HD214080 & 42.6 & 20 & 8.3 & 243.1 & 4 & 34 & no \\
\hline
\end{tabular}

References: (1) Ramspeck et al. 2001. (2) Tenjes et al. 2001. (3) Conlon et al. 1992. (4) Keenan et al. 1986. (5) Keenan et al. 1984.

last column. Note that the stars from Ramspeck et al. have galactic orbits calculated by the authors with the Allen-Santillán galactic potential, and the results quoted by them are, in fact, very similar to those we obtain. However, Ramspeck et al. give as "ejection velocities" the galactocentric velocity the star attains when reaching the plane. We think the ejection velocities should be referred to the local circular velocity, since it is that velocity which is typical for the parent clusters. This is the velocity we give in Tables 1 and 2 for all stars.

One further point deserves mention. Two of the stars in Table 2, namely HI 060350 and BD -2 3766 have velocities which exceed the local escape velocity; in other words, if we take their data at face value, they would not be bound to the Galaxy. The probability of this occuring is, however, exceedingly small (see discussion in Allen, Martos and Poveda 1987), and we think it is more likely that the distances to these stars have been overestimated. Both stars have orbits that are marginally bound to the galaxy when run with the errors added or subtracted.

The stars of Table 2, coming as they do from a variety of sources, have not been studied spectroscopically in a homogeneous way. We have seen that for at least two of them the distances are likely to be overestimated. For these reasons, we consider the stars of Table 2 to be more likely to harbour errors, and have dealt with them separately.

There are data in the literature for another 30 or 40 early stars far from the plane, though many lack proper motion information. We are in the process of studying the limits to the dynamical times-of-flight that can be estimated for these stars with the limited data available.

\section{DISCUSSION AND CONCLUSIONS}

We have shown that the dynamical ejection mechanism is a plausible alternative to explain the majority of the stars in Tables 1 and 2. However, for 8 stars of Table 1 and 4 of Table 2 the times of flight are larger than the nuclear lifetimes, and these stars do not have time to reach the $z$-distances at which they are observed. We could pose the question as to whether there are ways to prolong the nuclear lifetimes of massive stars. One obvious possibility is rapid rotation, which will induce mixing. However, models calculated with rotation increase the nuclear lifetimes by at most 20 percent (Meynet and Maeder 2000 ). So, rotation would solve the problem only for three additional stars of Table 1 and for 2 from Table 2 . They are marked by an ' $r$ ' in the last column of Tables 1 and 2.

Another possibility we can explore is star formation not on the galactic plane, but a few hundreds of parsecs above or below it. Such a mechanism was proposed by Martos et al. (2000), and is a result of the passage through the disk of a spiral density wave, which can eject sheets of gas to distances of up to $800 \mathrm{pc}$ from the plane. After the spiral density wave passes, the gas will fall back onto the plane; however, in certain cases, Martos et al. showed that conditions are favorable for star formation while the ejected gas is still far from the plane. We can en- 
visage that, as occurs in the plane, star formation will result not in single stars being born, but rather multiples or small clusters, within which the dynamical ejection mechanism could take place. We would then have runaway stars being produced not at $z=$ 0 but at $z=500-800 \mathrm{pc}$.

Returning to the orbital analysis, we can determine the times of flight not since the star left the plane, but since the star left a region situated 700 pc above or below the plane, where it could have formed according to the Martos et al. scheme. Such times of flight are, of course, shorter than the times of flight from the plane, and could be shorter than the nuclear lifetimes of the problem stars. The stars for which this is the case are marked by an ' $\mathrm{f}$ ' (for birth far from the plane). There is a total of five stars from Table 1 and two stars from Table 2 for which formation away from the plane, as in the Martos et al. scheme, would make the ejection mechanism a plausible alternative.

To sum up our results, the last column of Tables 1 and 2 shows that taking into account both the increase in the nuclear lifetimes than results from stellar rotation and star formation away from the plane, 29 out of the 32 Conlon stars, that is, 91 percent, can be explained by the dynamical ejection mechanism. Also, 8 out of the ten additional stars $(80$ percent) can be so explained. So, out of a total of 42 young stars far from the galactic plane, the cluster ejection mechanism, or one of its variants, is able to explain 37 , or nearly 90 percent, leaving only 5 stars for which another explanation is necessary. Thus, the great majority of the young stars far from the galactic plane can be identified as runaway stars in the galactic halo.

\section{REFERENCES}

Allen, C., Martos, M., Poveda, A. 1987, Rev. Mex. Astron. Astrofis., 14, 213.

Allen, C., Santillán, A. 1993, Rev. Mex. Astron. Astrofis., $25,39$.

Conlon, E. S., Brown, P. J. F., Dufton, P. L., Keenan, F. P. 1989, A\&A, 224, 65.

Conlon, E. S., Dufton, P. L., Keenan, F. P., Leonard, P. J. T. 1990, A\&A, 236, 357.

Conlon, E. S., Dufton, P. L., Keenan, F. P., McCausland, R. J. H., Holmgren, D. 1992, A\&A, 400, 273.

Gies, D. R., Bolton, C. T. 1986, ApJS, 61, 419.

Keenan, F. P., Brown, P. J. F., Lennon, D. J. 1986, A\&A, $155,333$.

Keenan, F. P., Lennon, D. J. 1984, A\&A, 130, 175.

Lance, C. 1988, ApJS, 68, 463.

Leonard P. J. T., Duncan, M. J. 1988, AJ, 96, 222.

Leonard P. J. T., Duncan, M. J. 1990, AJ, 99, 608.

Martos, M., Allen, C., Franco, J., Kurtz, S. 1999, ApJ, $525, \mathrm{~L} 89$.

Meynet, G., Maeder, A. 2000, A\&A, 361, 101.

Poveda, A., Ruiz, J., Allen, C. 1967, Bol. Obs. Tonantzintla Tacubaya, 4, 86.

Preston, G. W., Sneden, C. 2000, AJ, 120, 1014.

Ramspeck, M., Heber, U., Moehler, S. 2001, A\&A, 378, 917.

Ringwald, F. A., Rolleston, W. R. J., Saffer, R. A., Thorstensen, J.R. 1988, ApJ, 497, 717.

Rodgers, A. W., Harding, P., Sadler, E. 1981, ApJ, 244, 912.

Schaller, G., Schaerer, D., Meynet, G., Maeder, A. 1992, A\&AS, 96, 269.

Schmidt, J. H. K., de Boer, K., Heber, U., Moehler, S. 1996, A\&A, 306, L33.

Tenjes, P., Einasto, J., Maitzen, H.M., Zinnecker, H. 2001, A\&A, 369, 530.

Tobin, W. 1991, in IAU Symp. 144, The Interstellar DiskHalo Connection in Galaxies, ed. H. Bloemen, (Dordrecht: Kluwer), p.109.

Christine Allen: Instituto de Astronomía, Universidad Nacional Autónoma de México, Apartado Postal 70-264 04510, Mexico, D.F. (chris@astroscu.unam.mx).

T. D. Kinman: Kitt Peak National Observatory, National Optical Astronomy Observatory Operated by the Association of Universities for Research in Astronomy, Inc., under contract with the Nacional Science Foundation

Box 26732, Tucson, AZ 85726 - 6732, USA (kinman@noao.edu). 


\section{DISCUSSION}

Tokovinin - Some runaway stars are binaries. Did you check that the binding energy of those binaries is sufficient to survive ejection?

Allen - As far as I know, all binary runaways are spectroscopic binaries. As such, their binding energy is enough to survive dynamical ejection.

Zinnecker - Does not the ejection mechanism for runaway OB stars require dynamical interaction with a tight massive binary, and if so, would not runaway stars always come in a pair of stars one of which would necessarily be a binary?

Allen - Our mechanism for the formation of runaway stars (Poveda et al. 1967) requires close encounters within a multiple system ( 4 to 8 components). Energy and momentum are conserved by the formation of tight binaries and the recoil either of the remaining cluster or of a single runaway star escaping in the opposite direction.

Zinnecker - Are the properties of runaway O stars different from those of runaway B stars? What is the relative frequency of their occurrence?

Allen - Runaway stars are more frequent among the O-stars than among B stars. The frequencies given in the literature are of up to 20 for the $\mathrm{O}$ stars, and about 5

Clarke - Have you investigated where these runaways stars would have been ejected from in the Galactic plane, e.g., at locations coinciding with known OB associations?

Allen - No, we have not done that yet.

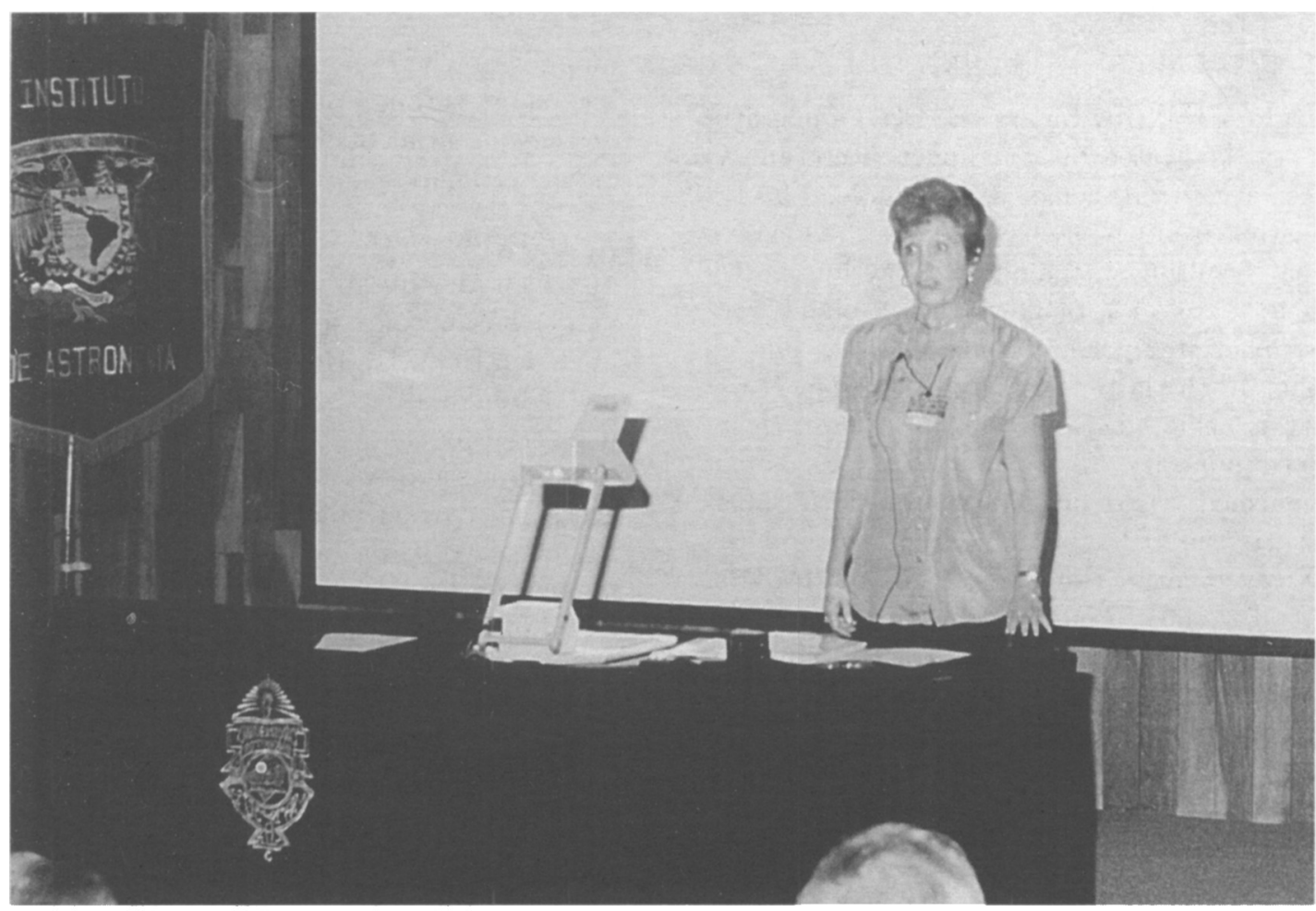

Christine Allen. 